This item was submitted to Loughborough's Research Repository by the author.

Items in Figshare are protected by copyright, with all rights reserved, unless otherwise indicated.

\title{
Fear and food: Effects of predator-derived chemical cues and stoichiometric food quality on Daphnia
}

PLEASE CITE THE PUBLISHED VERSION

https://doi.org/10.1002/Ino.11145

\section{PUBLISHER}

Wiley $@$ Association for the Sciences of Limnology and Oceanography

\section{VERSION}

AM (Accepted Manuscript)

\section{PUBLISHER STATEMENT}

This is the peer reviewed version of the following article: BELL, A.T.C. ... et al, 2019. Fear and food: Effects of predator-derived chemical cues and stoichiometric food quality on Daphnia. Limnology and Oceanography, 64 (4), pp.1706-1715, which has been published in final form at https://doi.org/10.1002/Ino.11145. This article may be used for non-commercial purposes in accordance with Wiley Terms and Conditions for Use of SelfArchived Versions.

\section{LICENCE}

CC BY-NC-ND 4.0

\section{REPOSITORY RECORD}

Bell, Alex T.C., Dennis L. Murray, Clay Prater, and Paul C. Frost. 2019. "Fear and Food: Effects of Predatorderived Chemical Cues and Stoichiometric Food Quality on Daphnia”. figshare.

https://hdl.handle.net/2134/37372. 
${ }^{1}$ Environmental and Life Sciences Graduate Program, Trent University, Peterborough, Ontario

$11{ }^{2}$ Department of Biology, Trent University, Peterborough, Ontario

12

13

14 Author contact information:

15 Corresponding author: A.T.C. Bell; albell@trentu.ca

16 Coauthors: P.C. Frost: paulfrost@trentu.ca; D.L. Murray: dennismurray@trentu.ca; C. Prater:

17 prater.clay@gmail.com

18

19

20

21 Keywords: Daphnia, ecological stoichiometry, food quality, indirect predator effects, life

22 history, phosphorus, predator-prey relationships

23 


\section{Abstract}

While resource quality and predator-derived chemical cues can each have profound

26 effects on zooplankton populations and their function in ecosystems, the strength and direction of

27 their interactive effects remain unclear. We conducted laboratory experiments to evaluate how stoichiometric food quality (i.e., algal carbon (C):phosphorus $(\mathrm{P})$ ratios) affects responses of the

29 zooplankter, Daphnia pulicaria, to predator-derived chemical cues. We compared growth rates,

30 body P content, metabolic rates, life-history shifts, and survival of differentially P-nourished

31 Daphnia in the presence and absence of chemical cues derived from fish predators. We found

32 effects of predator cues and/or stoichiometric food quality on all measured traits of Daphnia.

33 Exposure to fish cues led to reduced growth and increased metabolic rates but had little effect on

34 the body \%P content of Daphnia. Elevated algal C:P ratios reduced growth and body \% , and

35 increased mass-specific respiration rates. While most of the effects of predator cues and algal

36 C:P ratios of Daphnia were non-interactive, reduced survival and relatedly reduced population

37 growth rates that resulted from P-poor food were amplified in the presence of predator-derived

38 cues. Our results demonstrate that stoichiometric food quality interacts with anti-predator

39 responses of Daphnia, but these effects are largely trait-dependent and appear connected to

40 animal life-history evolution. Given the ubiquity of predators and P-poor food in lake

41 ecosystems, our results highlight the importance of the interactive responses of animals to

42 predator cues and poor nutrition. 


\section{Introduction}

The mere presence of predators can change the behavior, morphology, and life-history traits of prey (Stibor 1992; Tollrian 1995; Clinchy et al. 2013). In aquatic ecosystems, the presence of predators can be signaled through a variety of chemicals present in the water column, including kairomones, which are released directly by the predator, and alarm cues, which are released from prey species that have been attacked or alerted to the presence of predators (Pijanowska 1997; Dicke and Grostal 2001; Pestana et al. 2013). Prey have evolved complex behavioral, physiological, and demographic responses to these chemicals to reduce the risk of mortality due to predation (Lima 1998; Eklöv et al. 2006). The type and strength of such predator-induced responses and their subsequent effects on prey population dynamics can be influenced by environmental conditions, including food availability. Predator cues and the availability of food interactively affect prey reproduction (Pauwels et al. 2010), growth (DeLong and Walsh 2015), and behavior (Heithaus and Dill 2002). Recent work has also found variable stoichiometric responses in vertebrate prey exposed to predators (Costello and Michel 2013), especially when confronted with poor food quality (Dalton and Flecker 2014) and different environmental temperatures (Schmitz et al. 2016). Here we examine whether and how food quality affects the physiological responses of zooplankton exposed to predator cues and consequences on their population growth rates.

Low nutrient content in food results in elemental imbalances between zooplankton consumers, such as Daphnia, and their food resources (Sterner and Elser 2002). Elemental imbalances alter a range of physiological processes in consumers, including feeding rates, carbon assimilation, metabolic rates, and growth (Sterner and Elser 2002; Hessen et al. 2013). 
67 decreased body P content and reduced P excretion rates (DeMott et al. 1998), which is consistent with acute P-limitation. Similarly, animals consuming food having high C:P ratios alter C-based metabolic processes that appear to eliminate excess intake of C (Darchambeau et al. 2003; Frost et al. 2005). Due to constraints on animal energy and material budgets imposed by poor

71 elemental nutrition, prey may alter their physiological responses when exposed to predator cues 72 (Hawlena and Schmitz 2010; Dalton and Flecker 2014; Zhang et al. 2016). Poor stoichiometric 73 food quality could also affect key life-history responses in prey to predator exposure (Jeyasingh 74 and Weider 2005). Daphnia typically respond to chemical cues released from large bodied, 75 visual predators by reproducing earlier and at a smaller size, which increases reproductive 76 output, at least in younger population cohorts (Stibor 1992; Weider and Pijanowska 2003). These 77 life-history traits are also sensitive to poor stoichiometric food quality, which generally limits reproductive rates by increasing age of first reproduction and reducing brood sizes (Weider et al.

79 2008; Prater et al. 2016). Because poor stoichiometric food quality constrains reproduction, poorly-nourished prey may have reduced abilities to respond to perceived increases in predation

81 risk. When present together, the effects of predator cues and poor elemental food quality could 82 thus amplify or nullify the effects of each other and result in significant interactive effects. The responses of life-history traits of prey to environmental stress, both in terms of

84 predator exposure and poor elemental food quality, could translate into altered population growth rates. Population growth rates are a function of prey survival and reproduction as these rates

86 determine gains and losses of the prey population (Sibly and Hone 2002). As mortality rates 87 increase with the presence of predators, exposed prey generally respond by shifting to earlier reproduction (Stearns 1992; Stibor 1992). Predator-induced shifts in life-history traits may 89 require higher investments of resources at the time of first reproduction, which may be limited by 
nutrient-poor food (Zhang et al. 2016). If so, low nutrient food may constrain the earlier initiation of population growth, which can result from exposure to predator-derived cues. The nature of such interactive effects of predator cues and elemental food quality, if present, would depend of the relative strength and timing of effects on reproduction and animal mortality.

In this study, we examined whether and how predator-induced responses of Daphnia pulicaria are mediated by stoichiometric food quality. We did so by raising Daphnia in the laboratory in the absence and presence of cues released from juvenile bluegill (Lepomis

97 macrochrius), a common fish predator, and manipulating $\mathrm{C}: \mathrm{P}$ ratios in their algal food. We then measured physiological and life-history responses of Daphnia including mass-specific growth rates, metabolic rates, reproduction, and survival. We further used reproduction and survival data to examine population growth responses. We expected both increased diet $\mathrm{C}: \mathrm{P}$ ratios and exposure to predator cues to reduce growth rates, increase metabolic rates, and reduce animal survival. We also predicted that animals exposed to predator cues would optimize reproduction, 103 in terms of number and timing of offspring produced, brood size, and neonate size, but that these responses to predator exposure would be limited by higher $\mathrm{C}: \mathrm{P}$ ratios in the diet. Finally, we 105 predicted exposure to predator cues would reduce population growth rates and that the size of this reduction would increase with food C:P ratios.

\section{Methods}

Experimental design and treatments. We examined responses of differentially $\mathrm{P}-$ nourished Daphnia pulicaria to fish-predator cues. The daphnid clone used in this study was

111 originally collected from Big Cedar Lake in south-central Ontario (44.606638, -78.171669) and

112 cultured in the laboratory for $\sim 4$ years prior to use in our experiments. We collected juvenile 
113 bluegill (Lepomis macrochirus) from the Otonabee River near Peterborough, Ontario, Canada

$114(44.371780,-78.286718)$ to produce predator cues.

Daphnia brood mothers were reared in COMBO media (Kilham et al. 1998) and fed green algae (Scenedesmus obliquus, Canada Physiological Culture Centre, Strain 10). Neonates

117 ( $<24 \mathrm{hr}$ old $)$ were collected from the $2^{\text {nd }}-5^{\text {th }}$ broods of these mothers and randomly assigned to 118 different treatment combinations. For all experiments, Daphnia were reared individually in $50 \mathrm{ml}$ 119 plastic conical centrifuge tubes, which were maintained in a temperature-controlled growth 120 chamber $\left(20^{\circ} \mathrm{C}\right)$ and under low irradiance with a $14: 10$ light:dark photoperiod.

We measured several response variables during a series of full factorial $3 \times 2$ laboratory experiments where $D$. pulicaria were exposed to all six treatment combinations of food quality 123 (C:P 100, 300, and 600) and predator cues (absent or present). These algal C:P ratios were 124 selected to fit within the range previously reported for lake ecosystems (Hassett et al. 1997; Elser 125 et al. 2000a). To prepare the experimental diets, we cultured S. obliquus with varying media $\mathrm{P}$ 126 concentrations and dilution rates in multiple culture flasks. After harvesting, algal suspensions 127 were centrifuged for $10 \mathrm{~min}$ at $5000 \mathrm{rpm}$ and re-suspended in $\mathrm{N}$ - and $\mathrm{P}$-free COMBO media. 128 Algal P content was determined by persulfate digestion followed by molybdate-blue ascorbic acid colorimetry and spectrometry (APHA 1992), which allowed us to mix algae of different P contents to produce the three food $\mathrm{P}$ content levels. We determined the $\mathrm{C}$ and $\mathrm{N}$ content of algae with an elemental analyzer (Vario EL III, Elementar Incorporated, Mount Laurel, New Jersey).

132 The algal C:N:P ratios were determined on post-mixed food, using the same methods, to ensure 133 that the nominal food types were created.

Fish cues were collected from juvenile L. macrochirus $(80-110 \mathrm{~mm})$ housed for $24 \mathrm{hrs}$ in tanks with $4 \mathrm{~L}$ of $\mathrm{N}$ - and P-free COMBO media. The fish were fed live Daphnia during their 
136 incubation, so the media contained both predator kairomones as well as conspecific alarm cues.

137 The fish-conditioned media was then membrane filtered $(0.40 \mu \mathrm{m})$ and frozen at $-20^{\circ} \mathrm{C}$. Fresh

138 fish cues were harvested and frozen every four days, and before use in experiments the cue

139 media was thawed and diluted to a concentration equivalent to that produced by 0.1 fish L ${ }^{-1}$ day

140 . We selected this concentration of the predator cue based on reported concentrations from past

141 experiments that also examined Daphnia responses to fish-predators (Pauwels et al. 2010;

142 Tollrian et al. 2015). While the natural relevance of this concentration is unclear due to

143 differences between lakes and laboratory bottles (e.g., Schindler et al. 1997), the selected fish

144 density (\#/L) is moderately higher than that reported for lake littoral zones (Mittelbach 1988).

145 Growth media was refreshed in all the experimental tubes every other day.

146 To account for the effects of additional P from the fish cue on food algal P content, we

147 determined the concentration of soluble reactive phosphorus (SRP) in the cue media prior to food

148 algae preparation. By using P concentration values from both the fish cue and algal food

149 cultures, we mixed food concentrations to the desired dietary C:P ratio $(100,300$, and 600$)$ under

150 the assumption that $100 \%$ of the fish derived-P would be acquired by the food algae.

$151 \quad$ Growth and body elemental content. We examined the effects of food C:P ratios and

152 predator cues on growth rates of $D$. pulicaria with a 6-day growth experiment. Age-synchronized

153 neonates were collected ( $<24 \mathrm{hr}$ old $)$, and subsets of these neonates were randomly assigned into

154 replicate tubes to be later used to quantify growth rates $(n=10)$ for each treatment combination.

155 Initial neonate mass was determined by collecting three subsets of 20 neonates, which were

156 transferred into pre-weighed aluminum tins, placed into a drying oven (at $\left.60^{\circ} \mathrm{C}\right)$ for $24 \mathrm{hr}$, and

157 reweighed with a microbalance. Experimental animals were reared in treatment conditions for 6

158 days and fed $4 \mathrm{mg} \mathrm{C} \mathrm{L}^{-1}$ of either C:P 100, 300, or 600 food every other day. These food rations 
were based on animal mass and feeding rates and provided food quantities in excess of those estimates of daily food requirements for fast-growing daphnids as previously verified in similar experiments (Wagner and Frost 2012). After 6 days of growth, all experimental animals were removed from tubes, rinsed with $\mathrm{N}$ and $\mathrm{P}$ free $\mathrm{COMBO}$, and dried for $24 \mathrm{hr}$. Mass-specific growth rates (MSGR) were calculated as:

$$
\operatorname{MSGR}=\frac{\ln (M 2)-\ln (M 1)}{\text { time }}
$$

where $\mathrm{M}_{2}$ is the final mass per Daphnia, $\mathrm{M}_{1}$ is the average initial neonate mass, and time is number of days of growth. Animals were saved for P analysis using the same methods as above on persulfate-digested animal bodies. The remaining animals were pooled and weighed in tin cups, which were then used to measure body $\mathrm{C}$ and $\mathrm{N}$ content; however, due to mortality issues and sample pooling that resulted in reduced sample size, the results of these analyses were not included here but can be found in our supplementary material (Supplementary material Appendix 1, Fig. A2). Metabolic rates. To assess metabolic responses of $D$. pulicaria, we measured rates of $\mathrm{O}_{2}$ consumption of 6-day old animals following the approach of McFeeters and Frost (2012). Animals were raised following the same procedure as outlined above. Small groups of Daphnia (3-6 individuals) were placed in respiration vessels $(0.5 \mathrm{ml})$ containing COMBO and the assigned algal food C:P ratio-fish cue treatment. The vessels were placed in a water bath $\left(21^{\circ} \mathrm{C}\right)$ and animals allowed to acclimatize for $10 \mathrm{~min}$. Oxygen consumption was recorded using a micro-oxygen probe (MRCh system; Unisense A/S; Aarhus, Denmark) for 10 min under low light to limit photosynthetic activity. For every five samples, two blank vessels (without Daphnia) were measured to determine the effect of algae and fish cues on oxygen concentrations; these baseline values were then used to correct oxygen consumption slopes for 
182 each treatment. These baseline changes in $\mathrm{O}_{2}$ concentration were relatively small $(\sim 1 \%)$ relative 183 to oxygen changes that occurred when Daphnia were in the vessels. After respiration

184 measurements were made, animals were collected, dried for $24 \mathrm{hr}$, and weighed. Mass-specific

185 respiration rates were calculated by dividing the average corrected oxygen consumption by both 186 total Daphnia mass and time of measurement.

Life table experiment. A 30-day laboratory life table experiment was conducted to investigate differences in life history trade-offs between animals fed different food C:P ratios and in the presence or absence of fish predator cues. For each treatment, 25 Daphnia were individually reared in tubes containing $30 \mathrm{~mL}$ (age 0-6 day) and then $40 \mathrm{~mL}$ (age 7-30 day) of $\mathrm{N}$-and P-free COMBO media. Animals were fed $4 \mathrm{mg} \mathrm{C} \mathrm{L}^{-1}$ of algal food every other day for the 192 first $6 \mathrm{~d}$ and then $8 \mathrm{mg} \mathrm{C} \mathrm{L}^{-1}$ for the rest of the experiment. These food rations were based on 193 animal mass and feeding rates and provided food quantities in excess of those estimates of daily 194 food requirements for fast-growing daphnids. Each tube was checked daily for animal mortality 195 and reproduction. We measured daily production of offspring, size at reproductive maturity,

196 brood size, and survival. Mortality and survival data were then used to calculate intrinsic rate of 197 increase (r) using Euler's equation,

$$
1=\sum_{x=0}^{n} l_{x} m_{x} \cdot \exp (-r x)
$$
where $\mathrm{x}$ is age (days), $1_{\mathrm{x}}$ is age-specific survivorship, and $\mathrm{m}_{\mathrm{x}}$ is age-specific fecundity.

200 Reproductive output $\left(R_{0}\right)$ was calculated by summing the product of $1 \mathrm{x}$ and $\mathrm{mx}$ from each 201 treatment on each day of the experiment. To measure differences in body size, 10 additional 202 animals of each treatment were reared simultaneously; photos were taken of each animal 203 immediately after the first clutch of neonates was released and then every $7 \mathrm{~d}$ for the rest of the 204 experiment. Body length measurements were made from the top of the eye to the base of the tail. 
Neonate experiment. To assess how food C:P ratios and fish cues affected neonate size, neonates were collected from the $1^{\text {st }}-3^{\text {rd }}$ broods of animals raised in treatments for 15 days. Ten replicate mother Daphnia were raised in each treatment; three neonates from each the first five reproducing animals from each brood were selected randomly for measurement. Photos were taken of the neonates and of the mothers for body length measurements. Daphnid mass was estimated with a length-mass power function which was originally developed from a $D$. pulicaria clone from a nearby lake (Prater et al. 2017). Mass-specific reproductive investment was calculated as neonate mass/mother mass $* 100$.

Statistical analyses. Effects of food C:P ratios and predator cues on MSGR, respiration, body $\%$ P, body size, and brood size were analyzed using 2-way ANOVA, with food C:P ratio and predator cue treatments used as fixed effects. Body size, size at first reproduction, neonate size, and reproductive investment were analyzed with a 2-way repeated-measures ANOVA using linear mixed-effect models with either individual or mother identity as random effects. The effect sizes from the 2-way ANOVA models were estimated using eta-squared $\left(\eta^{2}\right)$, which is a measure of the proportion of variance accounted for by each main effect and interaction term. Tukey's HSD was used to assess treatment differences for all parametric tests. Differences in survival were assessed using Cox proportional hazards regression models (Cox 1972), using food C:P ratios and predator cue treatments as covariates. The food $\mathrm{C}: \mathrm{P}$ failed to pass the assumption of proportional hazards, so the levels of each covariate were analyzed individually. To compare population parameters, 1000 values of $\mathrm{r}$ and $\mathrm{R}_{\mathrm{o}}$ were generated for each treatment combination using a jackknife technique (Meyer et al. 1986). As the residuals violated parametric assumptions of normality, they were statistically compared using a Kruskal-Wallis H test with Scheirer-Ray-Hare extension (Ashforth and Yan 2008, Prater et al. 2016), which is a non- 
parametric equivalent to a 2-way ANOVA. We used $\alpha=0.05$ and all data were checked for normality by visually assessing residuals of each model. Homogeneity of variances was assessed by plotting residuals of each model against fitted values. We used R statistical software (version 3.3.3 with R studio) for all statistical analyses and image analysis software (IMT i-Solution) for all daphnid body measurements.

\section{Results}

Growth and respiration. We found a significant interactive effect of food C:P ratios and predator cues on the mass-specific growth rates of $D$. pulicaria (Table 1 ). While this indicates that the response of growth rates to predator cues varied with food C:P ratios, this interactive effect $\left(\eta^{2}=0.04\right)$ was dwarfed by the main effects of slower growth produced by higher food C:P ratios $\left(\eta^{2}=0.36\right)$ and the presence of cues $\left(\eta^{2}=0.42\right.$, Fig. 1a). We also found effects of treatments on Daphnia body sizes over the span of the 30-day experiment; a food C:P and predator cue interaction was only found on the last day of the experiment, while relatively large main effects of both treatments $\left(\eta^{2}=0.13-0.63\right)$ were found on body sizes on animals aged 14,21 , and 30 days (Table 1, Supplementary material Appendix 1 Fig. A1). No significant interactive effects were found on respiration rates of Daphnia (Table 1, Fig. 1b). Respiration rates were elevated at the highest food C:P ratios and by exposure to fish cues (Table 1; Fig. 1b). We also did not detect significant interactive effects between food C:P ratios and predator cues on the body \%P content of Daphnia (Table 1); increasing algal C:P ratios reduced \%P in Daphnia, regardless of cue exposure (Fig. 1c).

Life history traits and survival. While there was no interactive effect of food C:P ratios and exposure to predator cues on the size at first reproduction of Daphnia (Table 1), this parameter was reduced in response to each treatment independently (Fig. 2a). In terms of neonate 
252 size, there was no food C:P ratio $\times$ cue interaction (Fig. 2b) but increasing food C:P ratios

253 consumed by Daphnia resulted in smaller offspring (repeated-measures: $F_{2,58}=37.55$, $p<0.001$ ).

254 There was an interactive effect of food C:P ratios and predator cues on mass-specific

255 reproductive investment, which was measured as neonate mass relative to maternal mass

256 (repeated-measures interaction: $\mathrm{F}_{2,297}=4.62$, $\mathrm{p}=0.011$; Fig. 2c). While this parameter increased in

257 response to cues, the size of this effect was larger for the food C:P ratio 100 and especially for

258 the food C:P ratio 600 (Fig. 2c). In our brood size analysis, we found an interactive effect

259 between our two treatments with strong effects of cues on animals consuming high food C:P

260 ratios. In these animals, exposure to predator cues increased the number of neonates born in the

261 first brood compared to that produced by unexposed animals (Table 1, Fig. 2d, Food C:P $\times$ Cue:

$\left.262 \eta^{2}=0.12\right)$. In contrast, predator cues had minimal influence on brood sizes of animals eating food

263 C:P ratios of 100 and 300 (Fig. 2d).

264 Cox regression showed food quality and cue exposure interacted to affect Daphnia

265 survival (Supplementary material Appendix 1, Table A1). Survival in food C:P 100 and 300

266 treatments was high (92-96\%) both in the presence and absence of predator cues (Fig. 3a,b). At

267 food C:P ratio 600, survival of non-cue exposed animals was reduced to approximately $50 \%$ of

268 animals at the end of the experiment. This effect in P-stressed animals was amplified by

269 exposure to fish cues, as we observed near population extinction ( $~ 96 \%$ mortality) of predator-

270 exposed animals fed P-poor algae over the 30-day experiment (Fig. 3c). Cox proportional hazard

271 models showed significant treatment effects on survival, but only in the food C:P 600 treatment.

272 In the absence of cues, the hazard ratio of animals fed food C:P 600 (HR=20.8, 95\% CI=2.7-

273 157.7) indicated that mortality risk increased by $\sim 20$ times relative to animals raised on high $P$.

274 When animals were raised on food C:P 600 and additionally exposed to predator cues, the 
275 estimated hazard ratio increased to 59.4 (95\% CI=7.9-445.5), meaning that animals exposed to

276 both cues and high food C:P ratios had $\sim 60$ times higher mortality risk relative to unexposed

277 animals consuming high food quality.

278 Reproductive output and population growth rates. There was no interactive effect of

279 treatments on reproductive output $\left(R_{0}\right.$, Table 2$)$. As food C:P ratios increased from 100 to 600 ,

280 there was a reduction of $R_{0}$ of over $85 \%$ in both cue treatment levels (Fig. 4a). Additionally,

281 exposure to predator cues led to reduced $R_{0}$ across all diet treatments (Table 2). Observed

282 differences in reproduction and survival translated into differences in the population-level

283 parameter of intrinsic rate of increase $(r)$, which is a measure of population growth and

284 organismal fitness. Food quality and cue treatments had significant interactive effects on $r$ (Table

285 2, Fig. 4b). In high-P conditions, $r$ was approximately $5 \%$ in higher in the fish cue treatment, but

286 as food C:P ratios increased, there was a 27\% reduction of $r$ of Daphnia exposed to predator

287 cues, relative to those in the absence of cues (Fig. 4b).

288

289 Discussion

290 Prey species face the difficult task of maximizing fitness in environments having variable

291 predator and nutritional conditions. This challenge is not trivial from a prey's perspective

292 because small changes in their reproduction and survival can yield substantial differences in

293 population growth rates (Frost et al. 2010; Storm and Lima 2010). Following this, prey that can

294 detect and respond to mortality risks should be at an advantage if this leads to altered life-history

295 traits, including reproduction, that account for changes in expected longevity. Such life-history

296 shifts, in terms of reproductive timing and effort, might only be observed in predator-exposed

297 prey if these responses are otherwise costly in the absence of high predator mortality risk 
298 (Tollrian 1995; Riessen 2012). Matching these expectations, prey modify their physiology, life299 history traits, and morphology when exposed to cues that signal predator presence and a higher risk of mortality (Stibor 1992; Beckerman et al. 2007). It remains unclear, however, whether and 301 how life-history responses to predator exposure would change with the nutritional state of prey 302 species.

From a physiological perspective, strong interactive responses to the combined effects of 304 predator cues and poor nutrition could emerge if these responses share a common energetic or 305 material basis. Yet, our individual-based measurements failed to match this prediction, as 306 interactive effects were weak or insignificant and animals exposed to fish cues grew slower, 307 respired faster, and exhibited smaller body size across all ages, regardless of diet. These main 308 effects of predator cues are consistent with previously documented patterns of prey life-history 309 shifts in response to size-selective predation (Stibor 1992; Gliwicz and Maszczyk 2007). In this 310 case, reduced growth rates and smaller body sizes may have resulted from energy and nutrients 311 being redirected away from biomass accumulation and used for other purposes. For example, the 312 maintenance of high body $\mathrm{P}$ in our study animals despite reduced growth rates produced by 313 exposure to predator cues suggests that P-rich RNA was instead used for reproduction (e.g., 314 oogenesis; Markow et al. 2001) or cellular maintenance functions (Bertram et al. 2006). It thus 315 appears that elemental food quality may not strongly modify or constrain physiologically-linked 316 prey responses to predator cues (Jeyasingh and Weider 2005).

317 Similar to life-history traits and body $\%$, we found no interactive effects of predator 318 cues and food quality on metabolic rates. While predator cues increased Daphnia respiration 319 rates in well-fed animals, no apparent effect of cues were observed in the most P-stressed 320 animals, who also exhibited elevated respiration rates in the absence of predator cues. It is 
321 unclear why cue exposure did not further increase mass-specific respiration rates beyond that

322 produced by poor food quality. While there may be an upper ceiling to elevated Daphnia

323 respiration rates that cannot be exceeded regardless of the combined presence of predator cues

324 and poor food quality, these effects may be partly mediated by direct treatment effects on animal

325 body mass. As our experiments were not designed to separate out the drivers (e.g., mass vs.

326 metabolic reshaping) of elevated mass-specific respiration, future work is needed to better

327 examine how nutrition and predator cues alter metabolic rates of Daphnia.

Predator cues are known to elicit changes in prey life-history traits related to reproduction

329 (Macháček 1991; Weider and Pijanowska 1993; Reede 1997). To compensate for lower lifetime

330 reproductive success resulting from reduced longevity, reproductive output can be increased in

331 younger Daphnia exposed to predator cues (Macháček 1991; Stibor 1992). We found evidence

332 of these changes, with interactive effects of predatory cues and food quality on daphnid mass-

333 specific reproductive investment and brood size. Daphnia invested more strongly in reproduction

334 when exposed to predator cues largely due to smaller maternal mass and invariant neonate

335 production. We found that this mass-specific reproductive investment increased the most in

336 animals consuming poor food quality, which indicates this predator cue induced response was

337 not eliminated with acute nutrient stress in Daphnia. The number of neonates in the first brood

338 was only increased in animals experiencing increased risk while consuming good food quality.

339 Otherwise, cues either had no effect (for food C:P ratios 100 and 300) or possibly reduced brood

340 size (food C:P ratio 600) on the first three broods. Consequently, despite changes in animal

341 growth and metabolism, exposure to predator cues yielded little effect on total reproduction. The

342 insensitivity of reproductive output $\left(R_{0}\right)$ to predator cue-food quality interactions further

343 indicates its importance even in animals experiencing nutritional and predator-induced stress. 
Mortality rates of Daphnia were modified by the presence of predator cues and by food quality. Highest survival occurred in animals consuming high-P food, regardless of predator treatment. Thus, shifting growth and timing of reproduction to younger instars did not appear to affect short-term survival ( 30 days) of well-nourished animals. While predator cues had minimal effects on survival of well-nourished prey, mortality in P-stressed animals increased dramatically with exposure to predator cues. Therefore, sustained P-stress appears to exacerbate trade-offs between sustaining reproduction and maintenance processes underlying survival (McNamara and Buchanan 2005).

While we found interactive effects of high food C:P ratios and predator cues on a number of physiological and life-history responses, it is unclear how these effects would change the ecological role of Daphnia in lake ecosystems. For example, in terms of respiration, predator exposure increased daphnid respiration only in well-nourished animals. These changes in respiration, if accompanied by increased feeding rates or altered feeding behavior, could alter Daphnia-phytoplankton dynamics at a whole-lake scale. Such changes may not appear in lakes with high food C:P ratios where increased respiration rates were found in Daphnia regardless of predator-cue exposure. In addition, it is unclear how interactive effects of food quality and predator cues on Daphnia population growth $(r)$ observed in this lab setting would translate to natural conditions in lakes. This uncertainty rests, in part, on the extent that mortality rates of Daphnia are higher in predator-rich lakes due to direct losses to predators beyond that generated simply by exposure to predators. In other words, higher mortality rates observed in P-stressed, predator exposed animals may be lower than those generated by predation itself. Alternatively, direct predator effects on nutrient-limited Daphnia populations may be over-estimated if individuals escaping consumption nevertheless experience high mortality from exposure to 
367 predator cues. To reduce this uncertainty, future work could focus on in situ zooplankton

368 population responses to predators and their cues in lakes with variable food quality and predator

369 densities.

370 For a parthenogenetic invertebrate such as Daphnia, the timing and quantity of early

371 reproduction can strongly drive population growth over relatively short, but environmentally

372 relevant ( 30 days), time periods (McCauley et al. 1996; Pestana et al. 2013). We found

373 population growth rates in well-nourished Daphnia increased modestly with exposure to predator

374 cues, which was driven primarily by higher reproductive investment, larger first brood sizes, and

375 high survival. This indicates that Daphnia feeding on high quality food while under threat of fish

376 predation compensate by accelerating reproduction (Zhang et al. 2016). This pattern was

377 opposite in P-deprived Daphnia, whose population growth rates were depressed by the presence

378 of fish cues. This reduced population growth rate corresponded with both smaller brood sizes

379 and remarkably higher mortality rates. It thus appears that stress generated by low P food limits

380 the ability of Daphnia to maintain early and increased reproduction without sacrificing survival

381 over subsequent time periods. Overall, our findings of strong interactive effects on survival and

382 population growth, despite limited evidence from individual-based parameters, highlight the

383 importance of using integrated parameters such as intrinsic growth rates for understanding the

384 effects of predators and food quality on prey dynamics in natural populations.

385 We have shown how predator cues and food C:P ratios interact to affect survival and

386 some aspects of reproduction, with significant consequences for Daphnia population growth

387 rates. As large differences in predation pressure and food quality occur in lakes across summer

388 growing seasons (Sommer et al. 2012), the interactive nature of these effects may combine to

389 influence population dynamics and biomass production in nature. A fuller understanding of these 
390 stoichiometric food quality effects on predator-induced responses will partly include deeper

391 knowledge of molecular underpinning of prey physiological changes (Wagner et al. 2013;

392 Mitchell et al. 2017). Such work could include studying how cue detection specifically translates

393 into changes in growth, reproduction, and metabolism. In addition, information on the elemental

394 requirements of predator cue responses at the biochemical level (Elser et al. 2000b) is necessary

395 to understand how poor food quality may constrain anti-predator life-history traits. Our results

396 also indicate that differences in reproduction and survival produced by predator cues may lead to

397 significant effects on population growth rate or organismal fitness. These life-history responses

398 should be incorporated into models of predator-prey interactions, including stoichiometrically-

399 explicit models (Elser et al. 2012; Leroux and Schmitz 2015), to better understand potential

400 impacts of these changes over multiple generations. Similar studies should be conducted on more

401 diverse taxa with other forms of nutrient limitation to determine the nature and ubiquity of

402 stoichiometric constraints on life-history responses to predation risk. Our results on an important

403 zooplankton consumer thus provide insights into a relatively understudied connection between

404 the bottom up effects of food resources and top down effects of predation and point to a need to

405 better connect predator-prey dynamics to emerging issues in nutritional ecology.

406

407 References

408 Ashforth, D., and N. D. Yan. 2008. The interactive effects of calcium concentration and

409 temperature on the survival and reproduction of Daphnia pulex at high and low food

$410 \quad$ concentrations. Limnol. Oceanogr. 53: 420-432. doi:10.4319/lo.2008.53.2.0420

411 APHA. 1992. APHA Method 3500-MG: Standard methods for the examination of water and

412 wastewater, 18th ed. In A. E. Greenberg, L. S. Clesceri and A. D. Eaton [eds.], American 
Public Health Associattion.

414 Ashforth,Beckerman, A. P., K. Wieski, and D. J. Baird. 2007. Behavioural versus physiological

415 mediation of life history under predation risk. Oecologia 152: 335-343.

416 doi:10.1007/s00442-006-0642-6

417 Bertram, S. M., J. D. Schade, and J. J. Elser. 2006. Signalling and phosphorus: correlations 418 between mate signalling effort and body elemental composition in crickets. Anim. Behav. 72: 899-907. doi:10.1016/j.anbehav.2006.02.012

420 Clinchy, M., M. J. Sheriff, and L. Y. Zanette. 2013. Predator-induced stress and the ecology of 421 fear. Funct. Ecol. 27: 56-65. doi:10.1111/1365-2435.12007

422 Costello, D. M., and M. J. Michel. 2013. Predator-induced defenses in tadpoles confound body 423

424

425 426 doi:10.2307/2985181

427 Dalton, C. M., and A. S. Flecker. 2014. Metabolic stoichiometry and the ecology of fear in 428 Trinidadian guppies: Consequences for life histories and stream ecosystems. Oecologia 176:

Cox, D. R. 1972. Regression models and life-tables. J. R. Stat. Soc. Ser. B 34: 187-220. 691-701. doi:10.1007/s00442-014-3084-6

Darchambeau, F., P. J. Faerøvig, and D. O. Hessen. 2003. How Daphnia copes with excess carbon in its food. Oecologia 136: 336-346. doi:10.1007/s00442-003-1283-7

DeLong, J. P., and M. R. Walsh. 2015. The interplay between resource supply and demand determines the influence of predation on prey body size. Can. J. Fish. Aquat. Sci. 7: 1-23. 
doi:10.4319/1o.1998.43.6.1147

437

438

439

440

441

442

443

444

445

446

447

448

449

450

451

452

453

454

455

456

457

458

Dicke, M., and P. Grostal. 2001. Chemical detection of natural enimeies by arthropods : An ecological perspective. Annu. Rev. Ecol. Syst. 32: 1-23. doi:10.1146/annurev.ecolsys.32.081501.113951

Eklöv, P., R. Svanbak, P. Eklo, and R. Svanba. 2006. Predation risk influences adaptive morphological variation in fish populations. Am. Nat. 167: 440-452. doi:10.1086/499544

Elser, J. J., W. F. Fagan, R. F. Denno, and others. 2000a. Nutritional constraints in terrestrial and freshwater food webs. Nature 408: 578-580. doi:10.1038/35046058

Elser, J. J., I. Loladze, A. L. Peace, and Y. Kuang. 2012. Lotka re-loaded: Modeling trophic interactions under stoichiometric constraints. Ecol. Modell. 245: 3-11. doi:10.1016/j.ecolmodel.2012.02.006

Elser, J. J., R. W. Sterner, E. Gorokhova, and others. 2000b. Biological stoichiometry from genes to ecosystems. Ecol. Lett. 3: 540-550. doi:10.1046/j.1461-0248.2000.00185.x

Frost, P. C., D. Ebert, J. H. Larson, M. A. Marcus, N. D. Wagner, and A. Zalewski. 2010. Transgenerational effects of poor elemental food quality on Daphnia magna. Oecologia 162: 865-872. doi:10.1007/s00442-009-1517-4

Frost, P. C., M. A. Evans-white, Z. V Finkel, T. C. Jensen, and V. Matzek. 2005. Are you what you eat? Physiological constraints on organismal stoichiometry in an elementally imbalanced world. Oikos 109: 18-28.

Gliwicz, Z. M., and P. Maszczyk. 2007. Daphnia growth is hindered by chemical information on predation risk at high but not at low food levels. Oecologia 150: 706-715. doi:10.1007/s00442-006-0528-7

Hassett, R. P., B. Cardinale, L. B. Stabler, and J. J. Elser. 1997. Ecological stoichiometry of N 
and $\mathrm{P}$ in pelagic ecosystems: Comparison of lakes and oceans with emphasis on the zooplankton-phytoplankton interaction. Limnol. Oceanogr. 42: 648-662. doi:10.4319/1o.1997.42.4.0648

Hawlena, D., and O. J. Schmitz. 2010. Herbivore physiological response to predation risk and implications for ecosystem nutrient dynamics. Proc. Natl. Acad. Sci. 107: 15503-15507. doi:10.1073/pnas.1009300107

Heithaus, M. R., and L. M. Dill. 2002. Food availability and tiger shark predation risk influence bottlenose dolphins habitat use. Ecology 83: 480-491. doi:10.2307/2680029

Hessen, D. O., J. J. Elser, R. W. Sterner, and J. Urabe. 2013. Ecological stoichiometry: An elementary approach using basic principles. Limnol. Oceanogr. 58: 2219-2236. doi:10.4319/1o.2013.58.6.2219

Jeyasingh, P. D., and L. J. Weider. 2005. Phosphorus availability mediates plasticity in lifehistory traits and predator-prey interactions in Daphnia. Ecol. Lett. 8: 1021-1028. doi:10.1111/j.1461-0248.2005.00803.x

Kilham, S. S., D. A. Kreeger, S. G. Lynn, C. E. Goulden, and L. Herrera. 1998. COMBO: A defined freshwater culture medium for algae and zooplankton. Hydrobiologia 377: 147159. doi:10.1023/A:1003231628456

Leroux, S. J., D. Hawlena, and O. J. Schmitz. 2012. Predation risk, stoichiometric plasticity and ecosystem elemental cycling. Proc. R. Soc. B Biol. Sci. 279: 4183-4191. doi:10.1098/rspb.2012.1315

Lima, S. L. 1998. Nonlethal effects in the ecology of predator-prey interactions. Bioscience 48: 25-34. doi:10.2307/1313225

Macháček, J. 1991. Indirect effect of planktivorous fish on the growth and reproduction of 
483

484

485

486

487

488

489

490

491

492

493

494

495

496

497

498

499

500

501

502

503

504

Markow, T. A., A. Coppola, and T. D. Watts. 2001. How Drosophila males make eggs: It is elemental. Proc. R. Soc. B Biol. Sci. 268: 1527-1532. doi:10.1098/rspb.2001.1673

McCauley, E., R. M. Nisbet, A. M. De Roos, W. W. Murdoch, and W. S. C. Gurney. 1996. Structured population models of herbivorous zooplankton. Ecol. Monogr. 66: 479-501.

Mcfeeters, B. J., and P. C. Frost. 2011. Temperature and the effects of elemental food quality on Daphnia. Freshw. Biol. 56: 1447-1455. doi:10.1111/j.1365-2427.2011.02586.x

McNamara, J. M., and K. L. Buchanan. 2005. Stress, resource allocation, and mortality. Behav. Ecol. 16: 1008-1017. doi:10.1093/beheco/ari087

Meyer, J. S., C. G. Ingersoll, L. L. Mcdonald, S. Marks, J. S. Meyer, C. G. Ingersoll, L. L. Mcdonald, and M. S. Boyce. 1986. Estimating uncertainty in population growth rates : Jackknife vs . bootstrap techniques. Ecology 67: 1156-1166.

Mitchell, M. D., K. R. Bairos-Novak, and M. C. O. Ferrari. 2017. Mechanisms underlying the control of responses to predator odours in aquatic prey. J. Exp. Biol. 220: 1937-1946. doi:10.1242/jeb.135137

Mittelbach, G. G. 1988. Competition among refuging sunfishes and effects of fish density on littoral zone invertebrates. Ecology 69: 614-623. doi:10.2307/1941010

Pauwels, K., R. Stoks, and L. De Meester. 2010. Enhanced anti-predator defence in the presence of food stress in the water flea Daphnia magna. Funct. Ecol. 24: 322-329. doi:10.1111/j.1365-2435.2009.01641.x

Pestana, J. L. T., D. J. Baird, and a. M. V. M. Soares. 2013. Predator threat assessment in Daphnia magna: The role of kairomones versus conspecific alarm cues. Mar. Freshw. Res. 64: 679-686. doi:10.1071/MF13043 
Pijanowska, J. 1997. Alarm signals in Daphnia? Oecologia 112: 12-16.

$$
\text { doi: } 10.1007 / \mathrm{s} 004420050277
$$

Prater, C., P. C. Frost, and N. D. Wagner. 2016. Effects of calcium and phosphorus limitation on the nutritional ecophysiology of Daphnia. Limnol. Oceanogr. 61: 268-278. doi:10.1002/lno.10208

Prater, C., N. D. Wagner, and P. C. Frost. 2017. Interactive effects of genotype and food quality on consumer growth rate and elemental content. Ecology 98. doi:10.1002/ecy.1795

Reede, T. 1997. Effects of neonate size and food concentration on the life history responses of a clone of the hybrid Daphnia hyalina x galeata to fish kairomones. Freshw. Biol. 37: 389396. doi:10.1046/j.1365-2427.1997.00167.x

Riessen, H. P. 2012. Costs of predator-induced morphological defences in Daphnia. Freshw. Biol. 57: 1422-1433. doi:10.1111/j.1365-2427.2012.02805.x

Schindler, D. E. and L. A. Eby 1997. Stiochiometry of fishes and their prey: Implications for nutrient recycling. Ecology, 78: 1816-1831. doi:10.1890/00129658(1997)078[1816:SOFATP $] 2.0 . \mathrm{CO} ; 2$

Schmitz, O. J., A. E. Rosenblatt, and M. Smylie. 2016. Temperature dependence of predation stress and the nutritional ecology of a generalist herbivore. Ecology 97: 3119-3130. doi:10.1002/ecy.1524

Sibly, R. M., and J. Hone. 2002. Population growth rate and its determinants: an overview. Philos. Trans. R. Soc. B Biol. Sci. 357: 1153-1170. doi:10.1098/rstb.2002.1117 Sommer, U., R. Adrian, L. De Senerpont Domis, and others. 2012. Beyond the Plankton Ecology Group (PEG) Model: Mechanisms Driving Plankton Succession. Annu. Rev. Ecol. Evol. Syst. 43: 429-448. doi:10.1146/annurev-ecolsys-110411-160251 
Stearns, S. C. 1992. The Evolution of Life Histories, Oxford University Press.

Sterner, R. W., and J. J. Elser. 2002. Ecological stoichiometry: the biology of elements from molecules to the biosphere, Princeton University Press.

Sterner, R. W., and K. L. Schulz. 1998. Zooplankton nutrition: Recent progress and a reality check. Aquat. Ecol. 32: 261-279. doi:10.1023/A:1009949400573

Stibor, H. 1992. Predator Induced Life-History Shifts in a Freshwater Cladoceran. Oecologia 92 : 162-165. doi:10.2307/4220144

Storm, J. J., and S. L. Lima. 2010. Mothers forewarn offspring about predators: A transgenerational maternal effect on behavior. Am. Nat. 175: 382-390. doi:10.1086/650443

Tollrian, R. 1995. Predator-induced morphological defences: cost, life history shifts, and maternal effects in Daphnia pulex. Ecology 76: 1691-1705. doi:10.2307/1940703

Tollrian, R., S. Duggen, L. C. Weiss, C. Laforsch, and M. Kopp. 2015. Density-dependent adjustment of inducible defenses. Sci. Rep. 5: 12736. doi:10.1038/srep12736

Wagner, N. D., and P.C. Frost. 2012. Responses of alkaline phosphatase activity in Daphnia to poor nutrition. Oecologia 170: 1-10.

Wagner, N. D., H. Hillebrand, A. Wacker, and P. C. Frost. 2013. Nutritional indicators and their uses in ecology. Ecol. Lett. 16: 535-544. doi:10.1111/ele.12067

Weider, L. J., P. D. Jeyasingh, and K. G. Looper. 2008. Stoichiometric differences in food quality: Impacts on genetic diversity and the coexistence of aquatic herbivores in a Daphnia hybrid complex. Oecologia 158: 47-55. doi:10.1007/s00442-008-1126-7

Weider, L. J., and J. Pijanowska. 1993. Plasticity of Daphnia life histories in response to chemical cues from predators. Oikos 67: 385-392. doi:10.2307/3545351

Wisenden, B. D. 2000. Olfactory assessment of predation risk in the aquatic environment. Philos. 
Trans. R. Soc. B Biol. Sci. 355: 1205-1208. doi:10.1098/rstb.2000.0668

552 Zhang, C., M. Jansen, L. De Meester, and R. Stoks. 2016. Energy storage and fecundity explain 553 deviations from ecological stoichiometry predictions under global warming and size-

$554 \quad$ selective predation. J. Anim. Ecol. 85: 1431-1441. doi:10.1111/1365-2656.12531

555

556 Acknowledgements

557 We would like to thank Elyse Wakeford for her outstanding assistance with Daphnia husbandry 558 and laboratory experiments. We further thank both Thomas Hossie and Elizabeth Kierepka for 559 their statistical advice. Funding was provided to PCF and DLM by the Natural Sciences and 560 Engineering Research Council of Canada.

561

562 
564

565 Table 1: Main and interactive effects of food C:P ratios and fish cues on Daphnia traits, using

566 two-way ANOVAs. $\eta^{2}$ values estimate the proportion of error associated with main effects and

567 interaction terms, out of total variation. Significant effects are in bold.

568

Food C:P

$\begin{array}{llll}\text { df } & \eta^{2} & \text { F } & p\end{array}$

Cue

$\begin{array}{llll}\text { df } & \eta^{2} & \text { F } & \text { p }\end{array}$

Food C:P X Cue

$\begin{array}{llll}\text { df } & \eta^{2} & \text { F } & p\end{array}$

$\begin{array}{lllllllllllll}\text { MSGR } & 2,53 & 0.36 & 53.9 & <\mathbf{0 . 0 0 1} & 1,53 & 0.42 & 126.0 & <\mathbf{0 . 0 0 1} & 2,53 & 0.04 & 6.51 & \mathbf{0 . 0 0 3}\end{array}$

Body size

$\begin{array}{lllllllllllll}\text { Day } 14 & 2,44 & 0.64 & 94.4 & <\mathbf{0 . 0 0 1} & 1,44 & 0.12 & 61.4 & <\mathbf{0 . 0 0 1} & 2,44 & 0.00 & 0.68 & 0.51\end{array}$

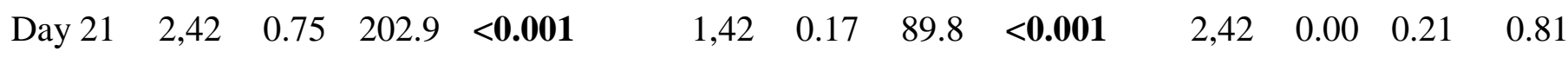

$\begin{array}{lllllllllllll}\text { Day } 30 & 2,38 & 0.71 & 129.6 & <\mathbf{0 . 0 0 1} & 1,38 & 0.17 & 61.3 & <\mathbf{0 . 0 0 1} & 2,38 & 0.02 & 3.56 & \mathbf{0 . 0 3 8}\end{array}$

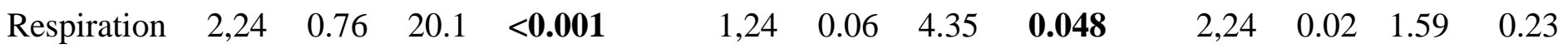

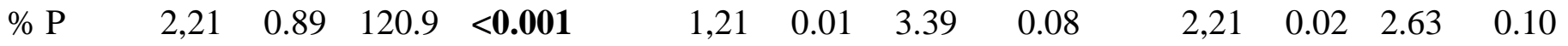

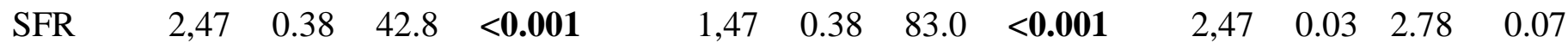

Brood size

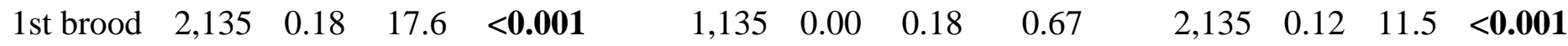

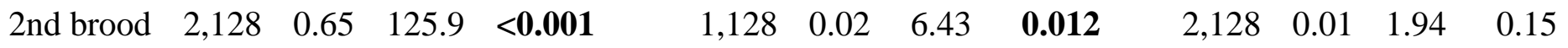

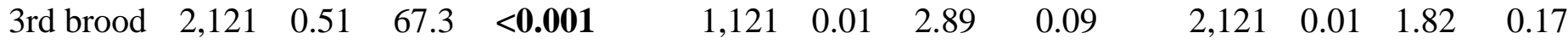

569 
571 Table 2: Main and interactive effects of food C:P ratios and fish cues on reproductive output $\left(R_{0}\right)$

572 and population growth rate $(r)$ using Kruskal-Wallis H test with Scheirer-Ray-Hare extension.

573 Significant effects are in bold.

\begin{tabular}{|c|c|c|c|c|c|c|}
\hline & \multicolumn{2}{|c|}{ Food C:P } & \multicolumn{2}{|c|}{ Cue } & \multicolumn{2}{|c|}{ Food C:P $\times$ Cue } \\
\hline & $\mathrm{H}$ & $\mathrm{p}$ & $\mathrm{H}$ & $\mathrm{p}$ & $\mathrm{H}$ & $\mathrm{p}$ \\
\hline$R_{0}$ & 5442.6 & $<0.001$ & 485.1 & $<0.001$ & 0.63 & 0.73 \\
\hline$r$ & 5379.9 & $<0.001$ & 52.4 & $<0.001$ & 444.9 & $<0.001$ \\
\hline
\end{tabular}

574

575

576

577

578

579

580

581

582

583 


\section{List of Figures}

585

Figure 1. (a) Mass-specific growth rates, (b) respiration rates, and (c) phosphorus body content

587 of Daphnia fed a gradient of food C:P ratios in the absence and presence of predator cues.

588 Asterisks indicate means that are significantly different within each diet treatment $(\mathrm{p}<0.05$,

589 Tukey’s HSD). Error bars show \pm 1 SE.

590

591 Figure 2. Effects of food C:P ratios and fish cues on (a) size of first reproduction, (b) neonate

592 size, and (c) mass-specific reproductive investment. Neonates used for analysis were collected

593 from the first 3 broods of mothers raised in each treatment combination. Mass-specific

594 reproductive investment was calculated as neonate mass/ mother mass *100. Bottom panels

595 show Daphnia brood size differences from the first 3 broods of animals fed algal food ratios of

596 (d) C:P 100, (e) C:P 300, and (f) C:P 600 and in the absence (open triangles) or presence (filled

597 triangles) of predator cues. Asterisks indicate means that are significantly different within each

598 diet treatment $(\mathrm{p}<0.05$, Tukey’s HSD). Error bars show $\pm 1 \mathrm{SE}$.

599

600 Figure 3. Effects of predator cues on survival of Daphnia fed algal food ratios of (a) C:P 100,

601 (b) C:P 300, and (c) C:P 600. Arrows indicate the relative timing of the first broods and dotted 602 lines show 95\% confidence intervals.

603

604 Figure 4. Effects of food C:P ratios and fish cues on (a) reproductive output $\left(R_{0}\right)$ and (b)

605 population growth rate $(r)$. Each point shows the mean and \pm 1 SD of values generated for each

606 treatment using a jackknife procedure. 
Figure 1.
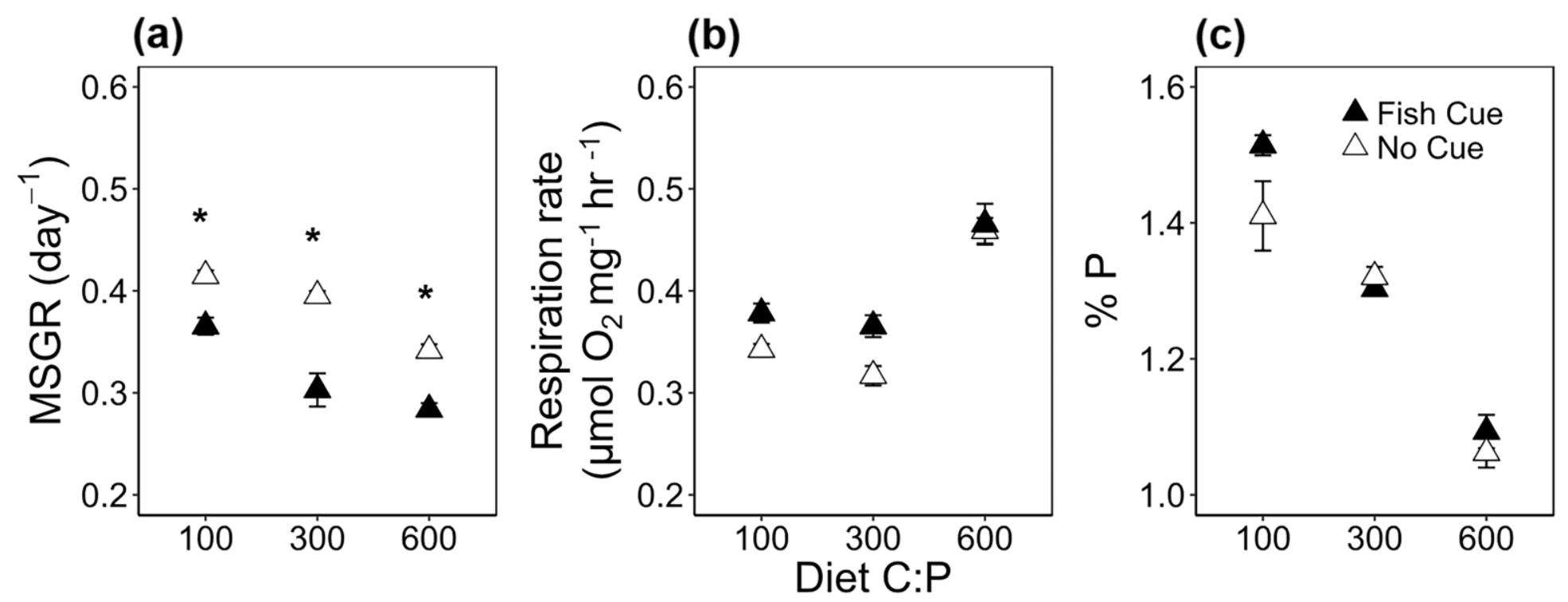
Figure 2.
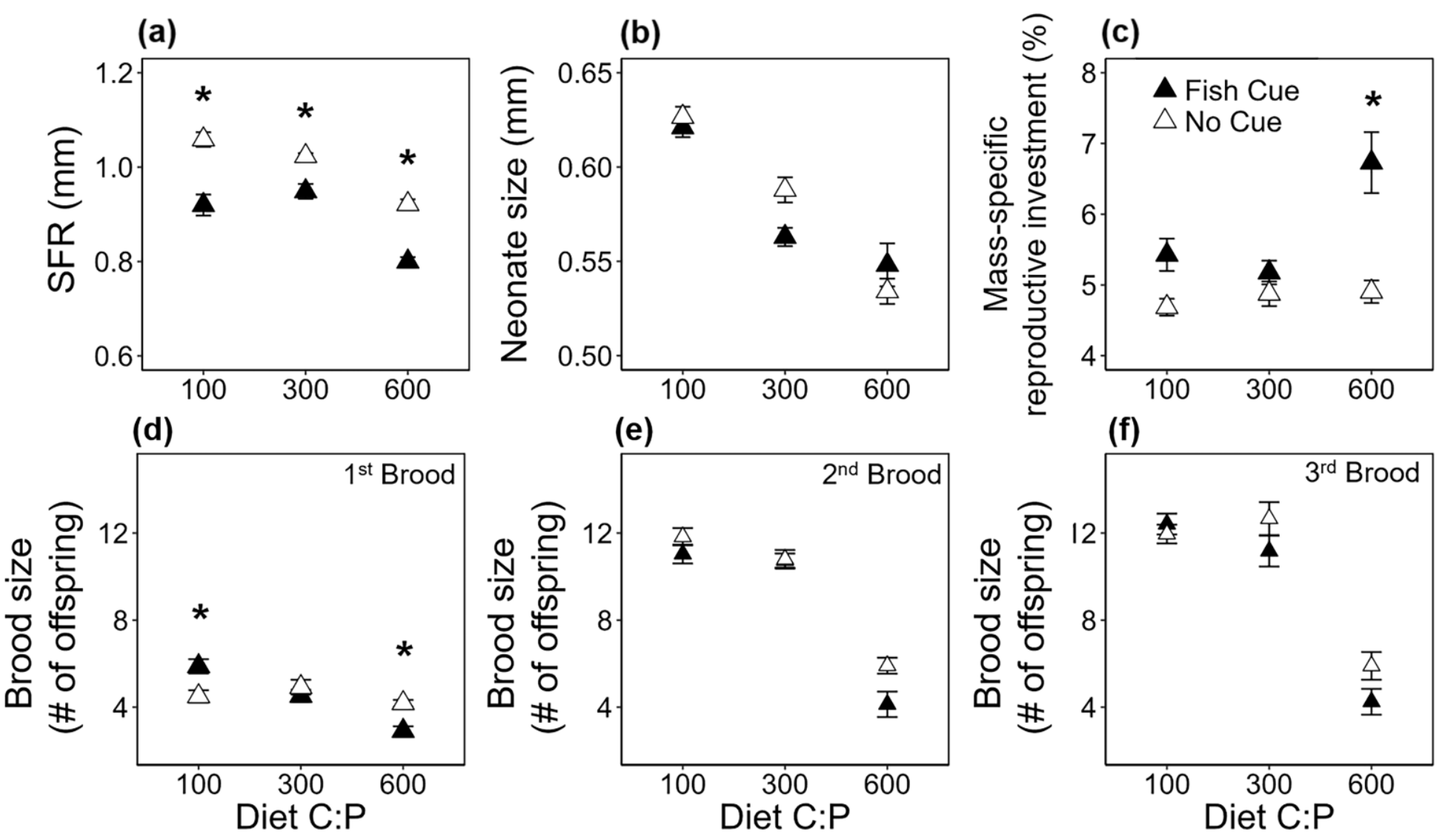
Figure 3.
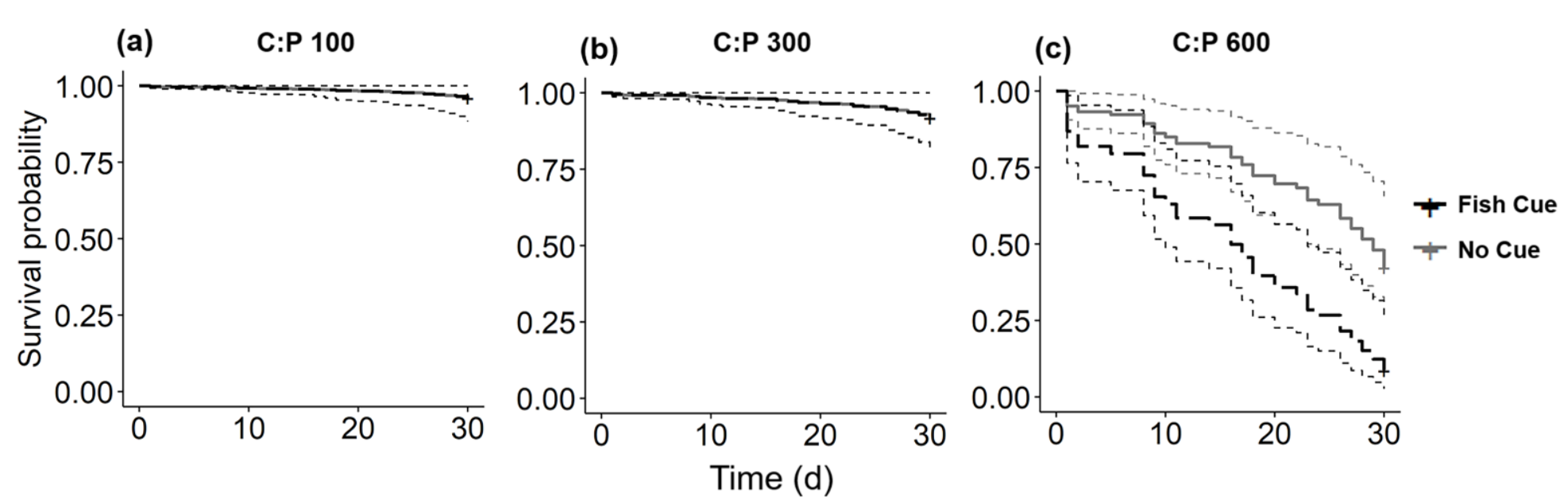
Figure 4.

(a)

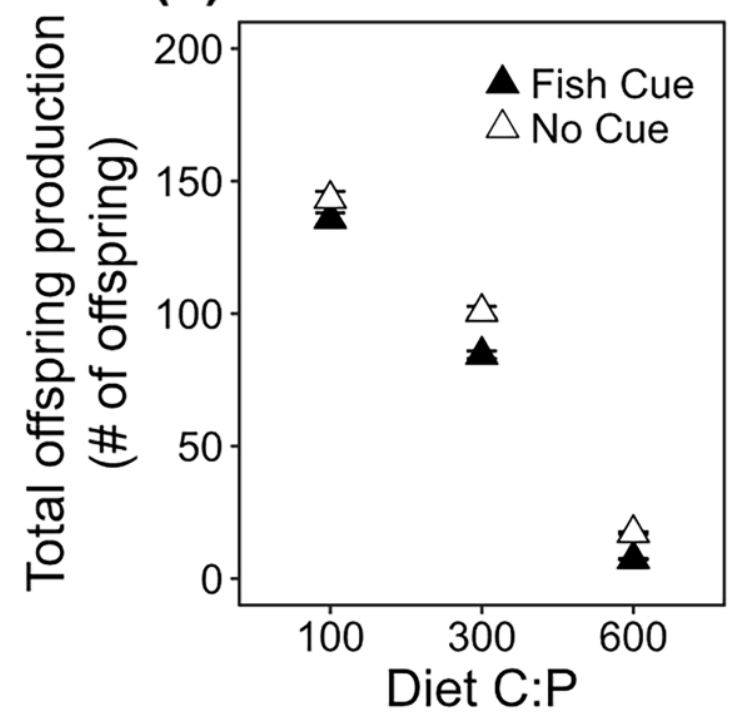

(b)

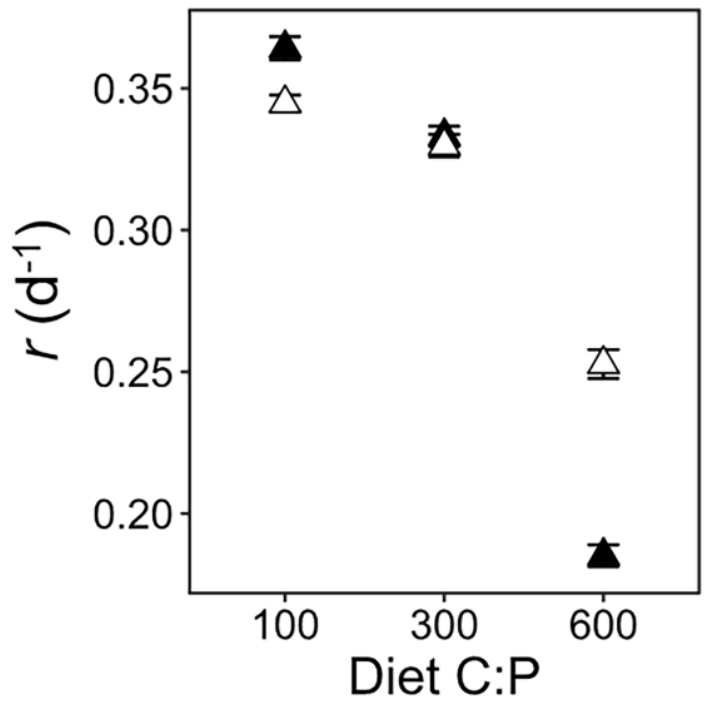

